
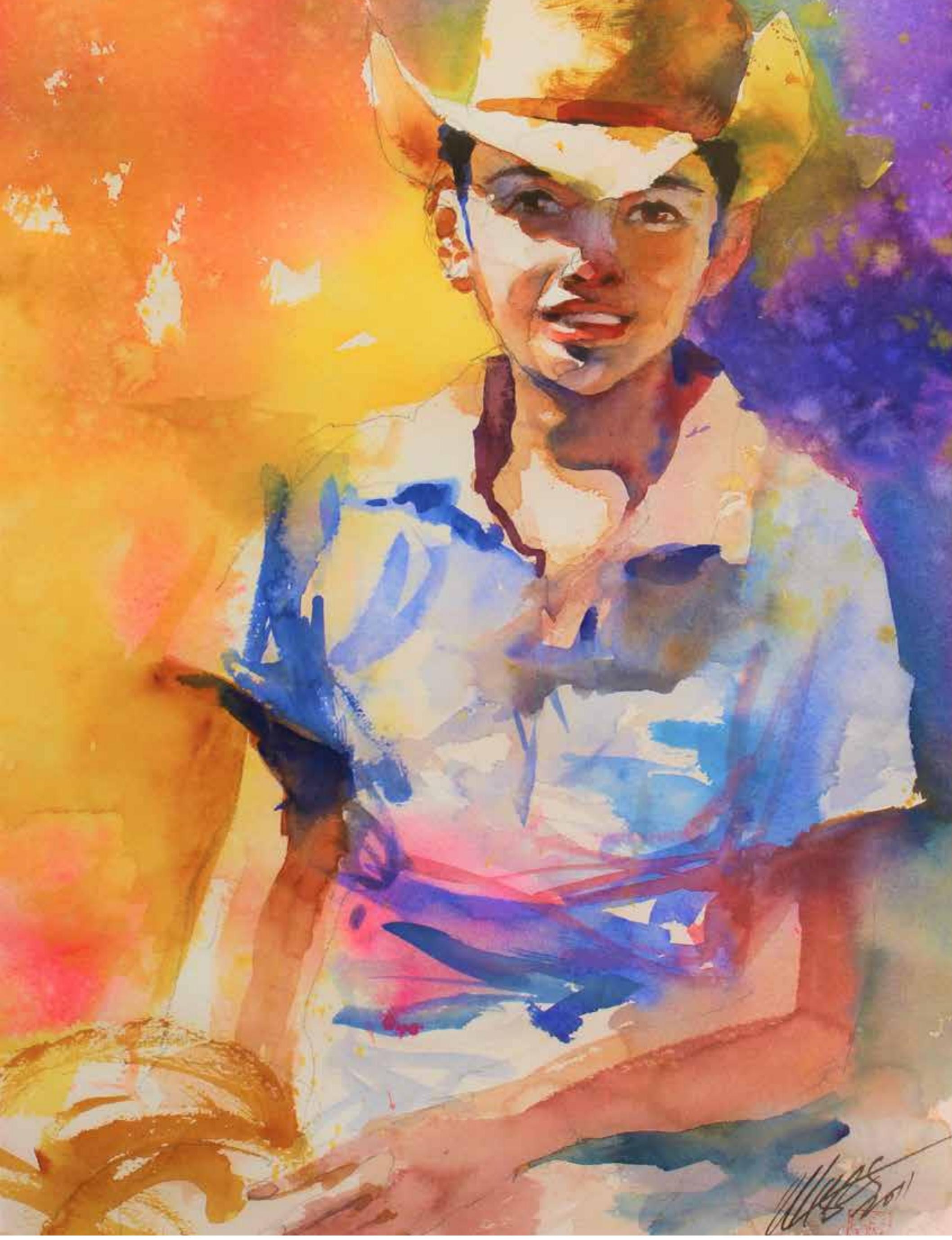


\section{Envejeciendo}

Cuando me veas sentada en silencio, Como un saco dejado en el estante,

No creas que necesito tu charla.

Me estoy escuchando a mi misma.

iEspera! iDetente! iNo te apiades de mi!

iEspera! iDetén tu simpatía!

Entendiendo si usted lo consiguió,

iDe lo contrario me haré sin él!

Cuando mis buesos estén entumecidos y doloridos,

$Y$ mis pies no suban la escalera,

Sólo te pediré un favor:

No me traigas una mecedora.

Cuando me veas caminando, tropezándome,

No estudiando y equivocándome.

Porque cansado no significa ser perezoso

Y cada adiós no se baya ido.

Soy la misma persona que fui entonces,

Un poco menos de pelo, un poco menos de barbilla,

Mucho menos pulmones y mucho menos aliento.

Pero sin suerte de que todavía pueda respirar.

Maya Angelou 


\title{
6. Las mujeres y el envejecimiento de la población total y activa de la Ciudad de Buenos Aires
}

Victoria Mazzeo'

Fabiana Bocchicchio ${ }^{2}$

DOI: https://doi.org/10.5377/pdac.v15i0.8119

Recibido: 06/05/2019 - Aceptado: 14/05/2019

Resumen: El envejecimiento de la población es un cambio de importancia que repercute en todas las facetas de la vida humana. El número de personas adultas mayores está aumentando, pero no es ese el problema sino el envejecimiento de la población activa lo que hace que una economía sea más vulnerable al debilitamiento de su crecimiento. La Ciudad de Buenos Aires presenta población envejecida desde 1960. En ese momento, la población de 65 años y más representaba ya 9,1\% y el proceso fue tan importante que, a partir de 1991, la sexta parte de su población se ubica en ese estrato. A partir del siglo XXI, los adultos mayores ya superan a los niños.

Se consideró interesante analizar la evolución del envejecimiento de la población en los últimos años y sus efectos en el mercado laboral de la ciudad. A partir de un abordaje cuantitativo, se indaga el crecimiento de los adultos mayores por sexo, tanto en la población potencialmente activa (PPA) como en la económicamente activa (PEA). El objetivo del artículo es responder la pregunta ¿Qué papel juegan las mujeres en el contexto del envejecimiento de la población activa en la Ciudad de Buenos Aires? La evidencia empírica muestra que el envejecimiento de la población se ha trasladado a la población activa y que el mismo está relacionado con el aumento de la PEA femenina.

Palabras clave: mujeres, envejecimiento, Población Total (PT), Población en Edad de Trabajar (PET), Población Económicamente Activa (PEA).

\section{Women and the ageing of the total and active population of the City of Buenos Aires}

\begin{abstract}
The aging of the population is an important change that affects all facets of human life. The number of older adults is increasing, but this is not the problem, but the aging of the active population that makes the economy more vulnerable to the weakening of its growth. The City of Buenos Aires has an aging population since 1960. At that time, the population of 65 years and over already represented $9,1 \%$ and the process was so important that from 1991, a sixth of its population is located in that stratum. As of the 21 st century, older adults have outgrown children.
\end{abstract}

The intention of this article is to analyze the evolution of the aging of the population in recent years and its effects on the city's labor market. Based on a quantitative approach, the growth of older adults is investigated by sex, both in the potentially active population (PPA) and in the economically active population (PEA). Finally yet importantly, the objective of this article is to answer: What role do women play in the context of the ageing of the active population in the City of Buenos Aires? The empirical evidence shows that the ageing of the population has been transferred to the active population and that it is related to the increase of the female PEA.

Keywords: women, ageing, Total Population (PT), Working Age Population (PET), Economically Active Population (PEA)

\section{Introducción}

Históricamente, el envejecimiento en las sociedades desarrolladas fue lento permitiendo adaptaciones sociales y económicas. En cambio, en los países en desarrollo este proceso está ocurriendo relativamente más rápido, surgiendo como consecuencia problemas críticos de pobreza y desigualdad (Ham-Chande et al., 2009).

Por este motivo, el envejecimiento demográfico ha pasado a ser una temática relevante en la investigación sociodemográfica de América Latina, y se le trata como un tema de actualidad. Las poblaciones envejecen por el aumento de la participación de las cohortes que casi completas alcanzan edades avanzadas. En términos demográficos, alude al aumento absoluto y relativo de dicho grupo en la población total y a la prolongación de la vida que se expresa en los valores cada vez mayores, de la esperanza de vida al nacer y de los años por vivir de dicha población.

Se ha afirmado que la salida del mundo del empleo es lo que determina el ingreso a la edad de la vejez. La jubilación ha sido vista durante mucho tiempo como una "muerte

1 Profesora Titular Cátedra Demografía Social, Carrera de Sociología, Instituto Gino Germani, FSOC, UBA. e-mail: victoria.mazzeo@gmail.com 2 Profesora Adjunta Sociología del Trabajo, Carrera de Sociología e Instituto Gino Germani, FSOC, UBA. e-mail: fabianabocchicchio@gmail.com 
social" pero esta imagen pesimista de la jubilación ya no tiene vigencia hoy en día (Segalen, 2013).

La vejez se construye a lo largo de toda la vida. Los comportamientos de las variables sociales, económicas y demográficas están cambiando y las nuevas generaciones llegan con características propias de la modernidad (Pérez Díaz, 2003).

Estos cambios han influido sobre las condiciones de vida de las personas mayores; por lo tanto, se consideró interesante abordar la evolución del envejecimiento de la población en los últimos años y sus efectos en el mercado laboral de la ciudad. Se estima un insumo de interés crucial para el diseño de políticas de población que deberán considerar como crear las condiciones más favorables para la vida y el bienestar, en una sociedad que ha cambiado su estructura por edad y sexo.

El problema del envejecimiento de la fuerza de trabajo es un tema importante que convoca a un número cada vez mayor de países. Las estadísticas demográficas permiten constatar que, en los países más industrializados, es cada vez mayor el porcentaje de trabajadores que superan los 40 y 50 años de edad. La reconversión económica, la desocupación, la incorporación de nuevas tecnologías y los cambios organizacionales, ponen de relieve la elevada proporción de trabajadores involucrados en el fenómeno del envejecimiento. Es una noción eminentemente relativa que depende no sólo de la edad, sino también de las calificaciones profesionales, de la rama de actividad, de las tareas y del tiempo de trabajo (Laville, 1993).

El interés por analizar la estructura de la población económicamente activa potencial "se funda en la idea de que la estructura de edad es el determinante fundamental del perfil de experiencia de la población económicamente activa" (Comelatto, 2009, pág.24). Los cambios en la composición de la población están directamente relacionados con cambios en la estructura de edad de la oferta de trabajo. En este sentido, el envejecimiento implica que los trabajadores de mayor edad se vuelven relativamente más abundantes.

Estos cambios también afectarán las condiciones de inserción en el mercado de trabajo de los distintos grupos etarios. A su vez las mutaciones en las condiciones del mercado de trabajo (demanda y oferta de trabajo) se manifiestan en los distintos grupos de edad de dos maneras principales: en la participación económica y en los salarios relativos. Así, un aumento en la oferta relativa de trabajadores de un grupo etario determinado, se puede traducir en una caída del salario relativo y/o aumento de la tasa de desocupación y/o una caída en la participación económica de dicho grupo (Comelatto, 2009).
El futuro del mundo del trabajo en América Latina y el Caribe está marcado por dos grandes tendencias: el avance tecnológico y el envejecimiento poblacional. Ambos pueden provocar cambios en el crecimiento de la economía y la productividad, incrementar o disminuir la desigualdad y afectar al empleo y a los ingresos. Ante este escenario parece probable que las personas deban prolongar sus vidas activas y trabajar durante más años. Esto implica que los mercados deberán adaptarse para darles oportunidades de trabajo (BID, 2018).

\section{Metodología}

El objetivo del artículo es responder la pregunta: ¿Cuál es la importancia de las mujeres en el envejecimiento de la población activa de la ciudad? A partir de un abordaje cuantitativo, se muestra la feminización de la vejez y se indagan los cambios en la composición de la población en edad de trabajar y en la población económicamente activa en el siglo XXI.

Para analizar el crecimiento y envejecimiento de la población de la ciudad de Buenos Aires se hizo uso de las fuentes de datos de los censos nacionales y municipales (1855-2010) y la Encuesta Anual de Hogares (2002-2016). Se examinó la composición por sexo de la población total (PT), población en edad de trabajar (PET) y población económicamente activa (PEA).

Específicamente en el período 2002-2016, se explora el envejecimiento por grupos de edad y sexo de la población total, de la población en edad de trabajar y de la población activa. Los indicadores seleccionados para analizar su evolución son: índice de envejecimiento, composición de la población por grandes grupos de edad, edad mediana de la población y tasas de actividad y de empleo por tramos de edad y sexo.

Asimismo, para el último año, se comparan para dos universos (adultos mayores y resto de la población económicamente activa) otras características laborales: categoría ocupacional, asalarización, precarización, cuentapropismo, calificación, tecnología y horas semanales trabajadas.

\section{\|ll. Discusión de resultados}

\subsection{El envejecimiento de la población de la ciudad}

Entre 1855 y 1915 la Ciudad de Buenos Aires experimentó una verdadera explosión demográfica que la colocó entre las ciudades más populosas del mundo. A partir de este período se experimenta un crecimiento moderado hasta 1950, que la hizo alcanzar en 1947 una población cercana a los 3 millones de personas. Desde ese momento y hasta 
la actualidad se caracteriza por el estancamiento poblacional, registrando incluso en los períodos intercensales 1970-1980 y 1991-2001, tasas de crecimiento negativo (Lattes et al., 2010). La masiva inmigración de ultramar fue el componente demográfico que explica el extraordinario crecimiento de la población de la ciudad entre 1855 y 1915 (Gráfico $N^{\circ} 1$ ).

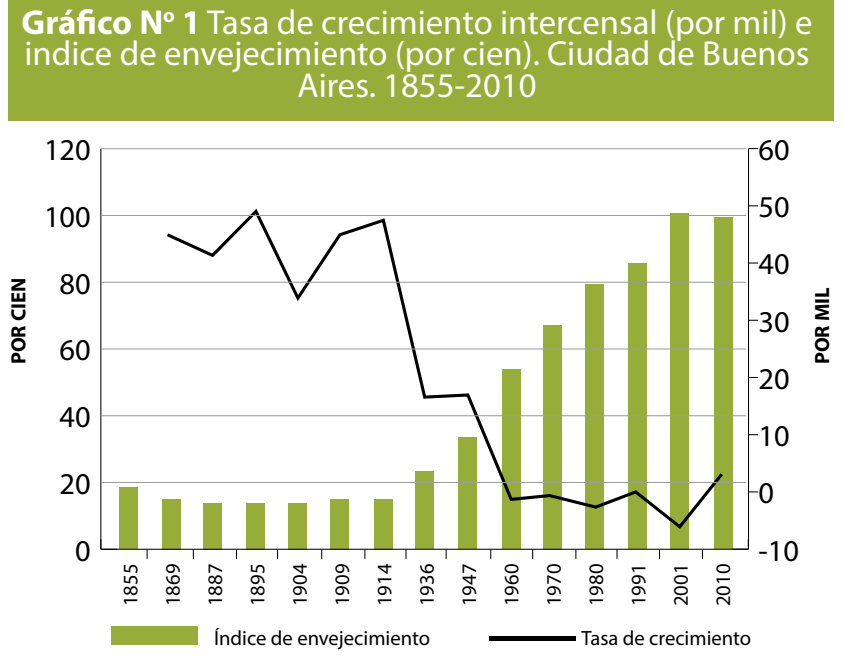

Fuente: Elaboración propia en base a datos censales.

Desde el punto de vista demográfico el proceso de envejecimiento de la población es el resultado del cambio en las tendencias de la fecundidad, de las migraciones y de la mortalidad. En las sociedades contemporáneas se encuentra relacionado al aumento numérico de la proporción de personas de 65 años y más. Se considera a una sociedad "envejecida" cuando esta proporción supera el $7 \%$ y en ese sentido puede decirse que la ciudad entró en esta categoría en la década de 1960, una vez completada la transición demográfica. En ese momento la población de 65 años y más representaba ya el $9.1 \%$ y el proceso fue tan importante que en 2010 la sexta parte de la población se ubicaba en ese estrato.

El índice de envejecimiento es un indicador del potencial de crecimiento de una población; es decir, su futuro reemplazo a través de la relación ancianos-niños (población de 65 años y más por cada cien personas de 0 a 14 años). Permite apreciar los cambios intergeneracionales derivados del proceso de envejecimiento. Estos ponen de manifiesto los cambios en las demandas sociales, sobre todo en materia de salud, $y$ en el sentido de las transferencias intergeneracionales. El índice aumenta progresivamente desde el año 1960 cuando alcanza un nivel cercano a 50 personas de 65 años y más por cien menores de 15 años hasta llegar a la paridad en el siglo XXI. De mantenerse las actuales condiciones no habría reemplazo en esa población, ya que los niños actuales no lograrían sustituir a los ancianos (Mazzeo, 2017).

El porcentaje de población de 65 años y más para ambos sexos creció en forma ininterrumpida de $9.1 \%$ al $16.4 \%$, se destaca la aceleración de su crecimiento a partir de los 70, especialmente en las mujeres, manteniendo una brecha respecto de los varones de 6 puntos porcentuales en promedio (Gráfico $\mathrm{N}^{\circ} 2$ ).

Gráfico № 2. Porcentaje de población de 65 años y más por sexo. Ciudad de Buenos Aires. 1960-2010

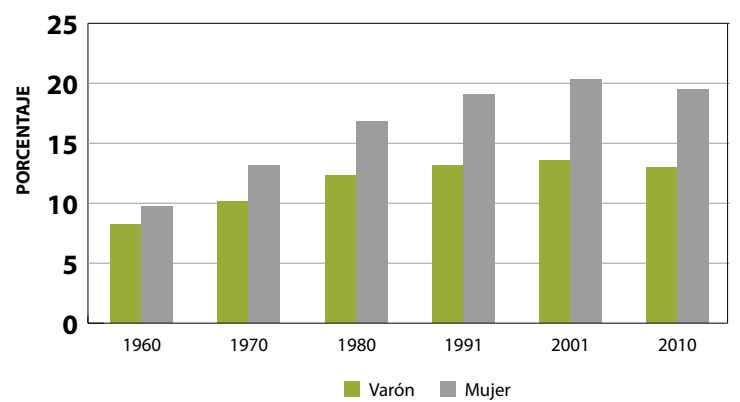

Fuente: Elaboración propia en base a datos censales.

La prolongación de la vida ocurrió de manera gradual durante el período analizado y su resultado se plasma en el sobre-envejecimiento de la población, es decir en la importancia creciente de las personas de 80 años y más. Entre 1960 y 2010 casi se quintuplica la participación de la "cuarta edad" en la población de la ciudad ( $1.1 \%$ en 1960 y $5.1 \%$ en 2010 ), principalmente en las mujeres (1.3\% y $6.6 \%$ respectivamente). Las tablas de mortalidad de 2008-2010 muestran que, en promedio, los varones de 65 años tienen por delante 16 años y a los 80 años poseen 7 años. Estos valores para las mujeres son más altos: 20 años y 9 años, respectivamente.

Ahora bien, este proceso ha sido acompañado por un progresivo envejecimiento de la población. Mientras que la población total entre 1960 y 2010 se redujo en cerca de 76,500 personas, los adultos mayores de 65 años y más aumentaron 1.7 veces y los de 80 años y más lo hicieron 3.6 veces.

Entre los años 1950 y 2000, tanto la población total como la PEA modificaron considerablemente su estructura por sexo y edad (Lattes et al., 2006a). La importante transformación estructural de la PEA se debió al gran aumento de la participación económica de las mujeres. Se identifican "dos etapas bien diferenciadas: 1950-1980 en el que la PEA total disminuye su tamaño y 1980-2000, en el que recupera y sobrepasa su tamaño inicial" (Lattes et al., 2006a, pág.71).

El aumento de la proporción de las mujeres en la PEA, entre 1980 y 2000, se relacionó con el desigual aumento de 
la actividad económica entre varones y mujeres; ellas lo hicieron en grado mucho mayor (Lattes et al., 2006b).

En síntesis, en Buenos Aires a partir del siglo XXI, los adultos mayores ya superan a los niños. Entre ellos se observa mayoría femenina, asociada a la sobremortalidad masculina y a la distinta composición de las migraciones que arribaron a la ciudad. La feminización de la vejez es evidente y a partir de las últimas décadas del siglo XIX creció su actividad económica. De ahí la importancia de evidenciar si la feminización del envejecimiento de la población total en el siglo XXI se trasladó al mercado de trabajo.

\subsection{El envejecimiento de la población en el siglo XXI y su re- lación con el mercado laboral}

El análisis de la evolución de la población total en la ciudad permite observar qué grupos etarios y de qué sexo crecieron más los quince años transcurridos entre 2002 y 2016. La variación porcentual de la población total entre dichos años fue del $14 \%$, más elevada entre los varones. Los menores de 10 años son los de mayor crecimiento. Respecto a la población en edad de trabajar el grupo 35-39 años es el de mayor aumento, especialmente entre los varones. No obstante, las mujeres lo superan entre los 30-34, 40-44, 60-64 y 70 años y más (Gráfico №3).

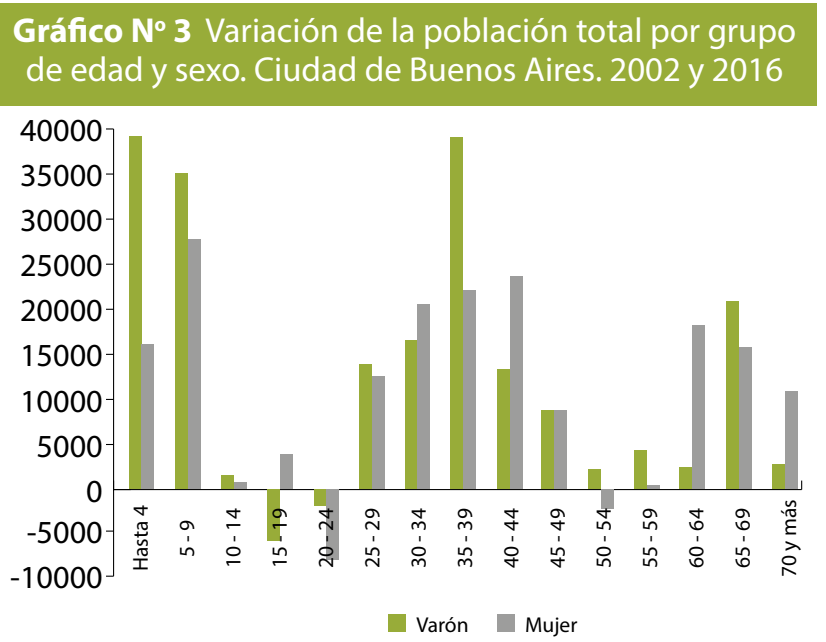

Fuente: Elaboración propia en base a datos de EAH 2002- 2016

Se destaca decrecimiento en los varones entre los 15 y 24 años y las mujeres de 20 a 24 años, quizás relacionados con los movimientos migratorios y la baja de la fecundidad continua de la ciudad. El crecimiento de las adultas mayores, muestra la notoria feminización del envejecimiento.

Ahora bien, el envejecimiento de la población total ¿cómo incidió en el mercado laboral? Para responder esta pregunta se investigó la composición porcentual de la población en edad de trabajar y la económicamente activa según grupo de edad y sexo y se comparó la evolución de las edades medianas de la población total (PT), en edad de trabajar (PET) y la económicamente activa (PEA) según sexo.

El envejecimiento de la población se ha trasladado a la Población en Edad de Trabajar (PET). Es necesario destacar la situación de los grupos extremos de edad. Entre los varones (Gráfico №4): los jóvenes han reducido su participación en $3.5 \%$ mientras que los mayores de 54 años la han aumentado en $5.9 \%$. En valores absolutos, se incrementaron en 95,000 y los de 55 años y más en 7,000 personas, mientras que los menores de 25 años se redujeron en 7,000 efectivos.

La situación entre las mujeres (Gráfico $\mathrm{N}^{\circ} 5$ ) es similar, entre las jóvenes (decrece el 1.5\%) pero es mucho más importante el aumento a partir de los 55 años, donde el volumen crece más del doble que en los varones. En valores absolutos, la PET femenina total crece 102,000 y en especial la de 55 años y más aumenta en 19,000 mujeres.

En los Gráficos №4 y №5 puede apreciarse claramente la evolución de la población en edad de trabajar entre 2002 y 2016 : notable descenso de la población en las cohortes de menor edad y destacado incremento en los tramos intermedio ( $35 \mathrm{a}$ 44 años) y de más edad (55 a 64 años).

\section{Gráfico N*4 Composición porcentual de los varones}

en edad de trabajar según grupo de edad. Ciudad de Buenos. 2002-2016

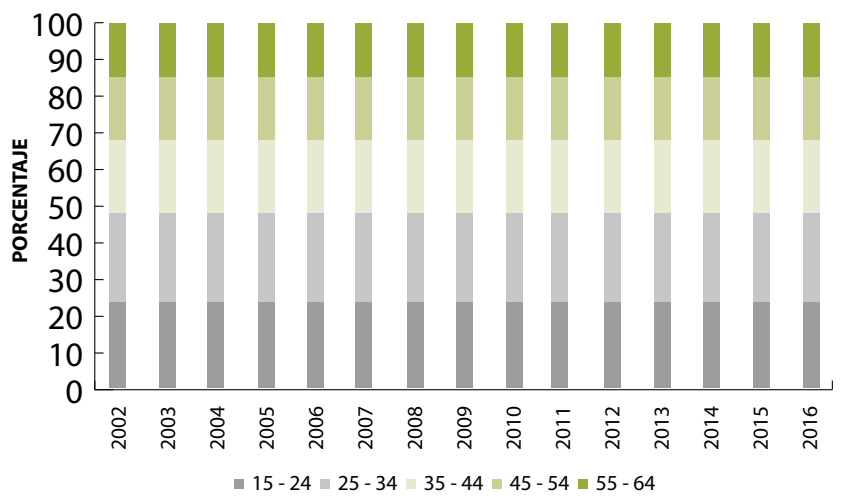

Fuente: Elaboración propia en base a datos censales.

Gráfico $\mathbf{N}^{\circ} 5$ Composición porcentual de las mujeres en edad de trabajar según grupo de edad. Ciudad de Buenos. 2002-2016

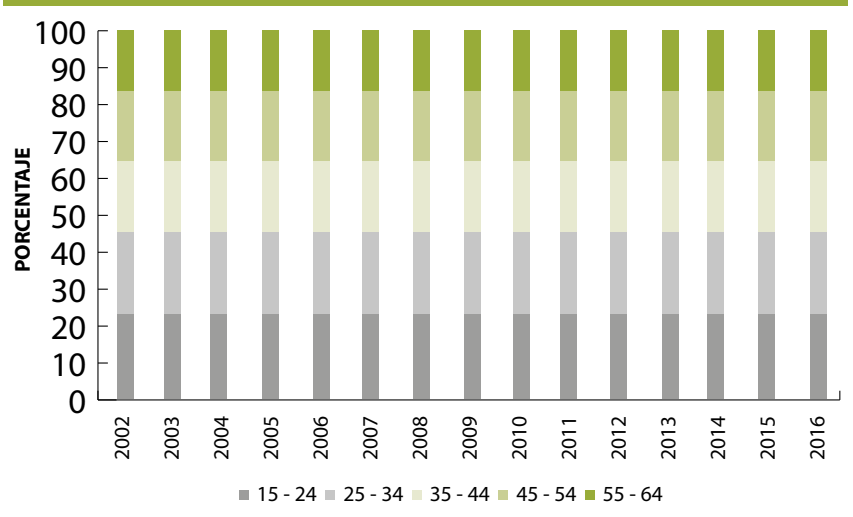

Fuente: Elaboración propia en base a datos censales. 
Ahora bien, ¿qué grupos crecieron más en la PEA y de qué sexo? Con respecto a los varones (Gráfico $\mathrm{N}^{\circ} 6$ ) los mayores crecimientos se registraron en: 65 y más (42\%), 35-44 (35\%) y 25-34 (19\%). La participación de los menores de 25 años decrece el 7\%. Por su parte en las mujeres (Gráfico № ${ }^{\circ} 7$ ) los grupos etarios que más crecen: 65 y más (76\%), 35-44 (43\%), 55-64 (38\%) y 25-34 (18\%). La participación de las menores de 25 años decrece el $16 \%$.

Gráfico № 6 Composición porcentual de la PEA masculina por tramos de edad. Ciudad de Buenos Aires. 2002-2016

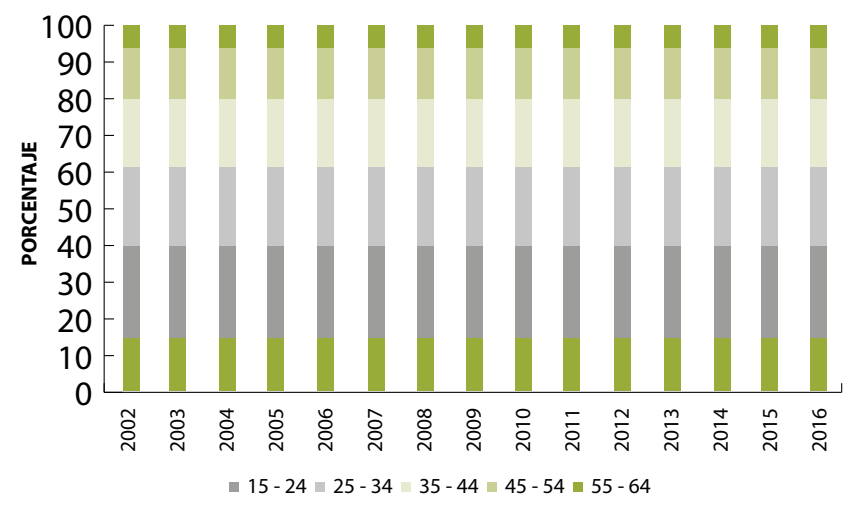

Fuente: Elaboración propia en base a datos EAH 2004-2016.

Gráfico $\mathbf{N}^{\circ} \mathbf{7}$ Composición porcentual de la PEA femenina por tramos de edad. Ciudad de Buenos Aires. 2002-2016

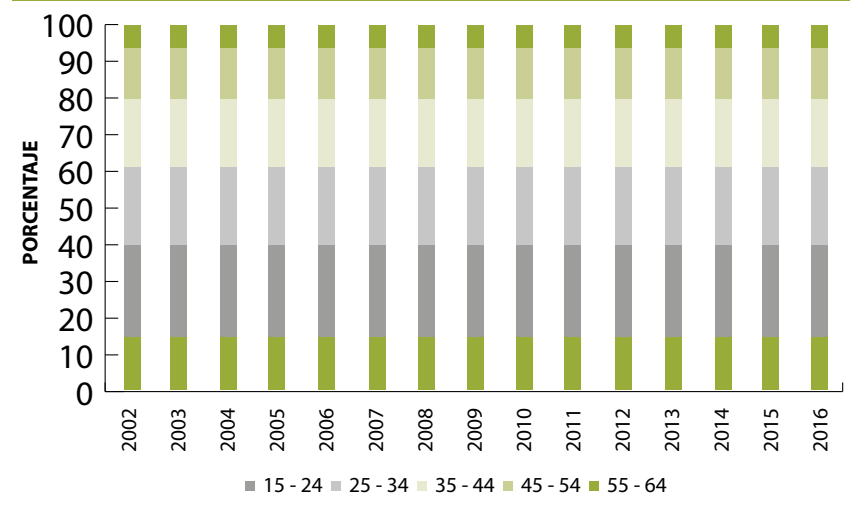

Fuente: Elaboración propia en base a datos EAH 2004-2016.

En valores absolutos, a la población activa entre 2002 y 2016, se incorporan más de 123,000 varones y más de 156,000 mujeres. A partir de los 25 años, en todos los grupos etarios se registran mayores aumentos de mujeres, las principales diferencias se ubican entre los 45 y 64 años donde el crecimiento femenino duplica al masculino
(56,000 mujeres más y 22,000 varones más). Por su parte, los menores de 25 años, decrecen en 26,000 efectivos, de los cuales los dos tercios son mujeres.

Además del envejecimiento poblacional, la PEA se ha visto condicionada por otros factores, entre los que cabe destacar: la creciente incorporación de la mujer al mercado de trabajo y los derivados de la crisis económica. Existe un alargamiento de la etapa formativa entre los jóvenes y una necesidad de prolongar la vida activa entre los adultos mayores. Cabe consignar que en 2016 cerca del 7\% de la fuerza de trabajo de la ciudad estaba constituida por personas de 65 años y más.

Otra manera de visualizar el envejecimiento de la PEA es a través de las edades medianas. En los trece años que se analizan, para los varones la edad promedio de la PT se mantiene en los 33 años (Gráfico $N^{\circ} 8$ ), la de la PET aumenta un año (35 a 36 años) y la PEA permanece en los 39 años. Entre la PET y la PEA la brecha se redujo de 4 a 3 años.

\section{Gráfico $\mathbf{N}^{\circ} \mathbf{8}$ Relaciones entre las edades medianas de la PT, PET y PEA masculina. Ciudad de Buenos Aires. 2004-2016}

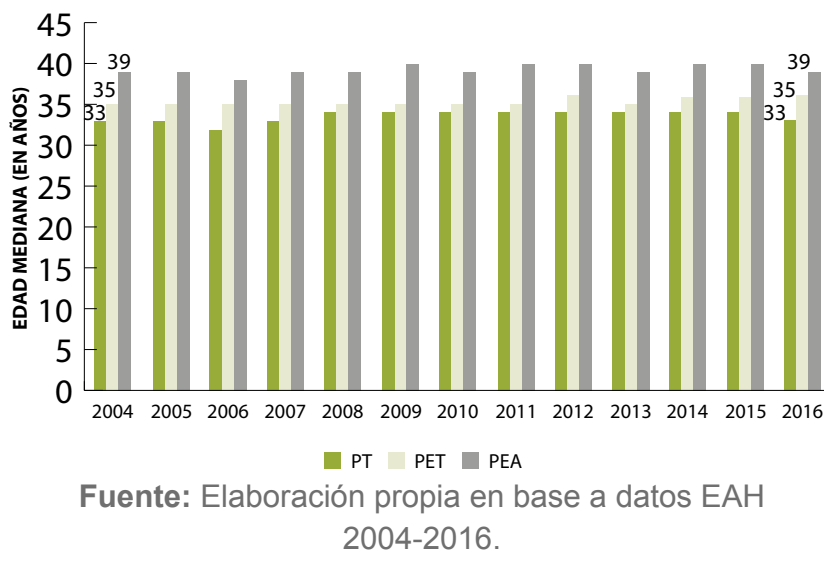

En las mujeres la edad promedio de la PEA es un año mayor (40 años) y tiene una brecha menor respecto a la PET (dos años). La mediana de la PT permanece en 38 años (5 años más que los varones). Estos valores muestran la existencia de la feminización del envejecimiento no sólo de la población total sino también de la población activa. Se destaca que en Argentina las mujeres pueden jubilarse a partir de los 60 años y los varones de los 65 años. 
Gráfico № 9 Relaciones entre las edades medianas de la PT, PET y PEA femenina. Ciudad de Buenos Aires. Años 2004-2016

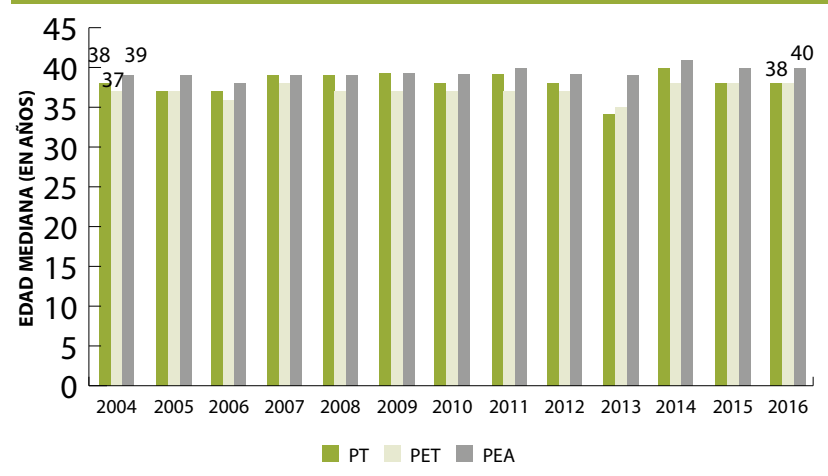

Fuente: Elaboración propia en base a datos EAH 2004-2016.

\subsection{Indicadores del mercado de trabajo en el siglo XXI}

Otro aspecto relevante a indagar es si la progresiva incorporación de la mujer al mercado laboral ha favorecido la tasa de actividad de la población a partir de los 15 años. Lo que hace necesario responder la siguiente interrogante: ¿Qué pasó con la actividad y el empleo por sexo? En este sentido, entre 2004 y 2016, se reduce la brecha entre las tasas de actividad y de empleo según sexo. En el Gráfico $N^{\circ} 10$ se destacan los mayores incrementos en las tasas de actividad (4 puntos porcentuales) y de empleo ( 3 puntos porcentuales) de las mujeres. Los varones, en el mismo período, sólo las aumentan en 1 punto porcentual.

Esto se relaciona con el incremento de las mujeres en el volumen de la PEA a partir de los 25 años, y especialmente después de los 45 años.

¿Qué pasó con las tasas de actividad por grupo de edad? En los menores de 25 años decrecen 3 puntos porcentuales (no debe olvidarse la importante reducción de efectivos en este grupo etario). En el resto de los grupos etarios aumenta entre 2 puntos porcentuales (75 y más) y 4 puntos porcentuales (50-64). En 2016 el 35\% del grupo 65-74 años registra estar ocupado o buscar activamente trabajo y este nivel decrece a $10.9 \%$ para los mayores de esa edad ${ }^{3}$ (Gráfico №11).

Esto demuestra que la progresiva incorporación de la mujer al mercado laboral habría favorecido el aumento de las tasas de actividad a partir de los 25 años. Por otro lado, muestran altos niveles de participación en una población que debiera estar gozando los beneficios de su jubilación.
Gráfico № 10 Tasas de actividad y de empleo (por cien) según sexo. Ciudad de Buenos Aires. Años 2004-2016

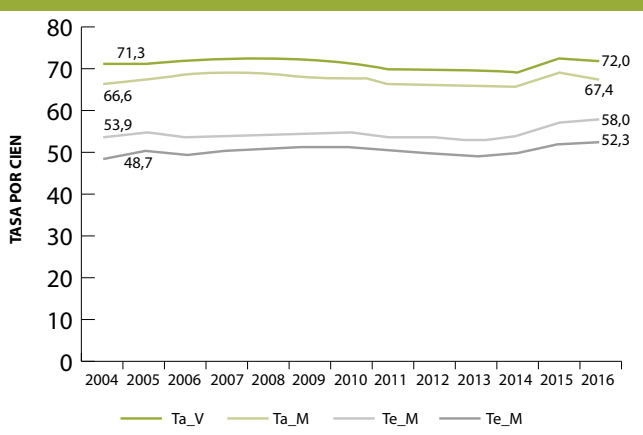

Fuente: Elaboración propia en base a datos EAH 2004-2016.

Gráfico № 11 Tasas de actividad (por cien) según grupo de edad. Ciudad de Buenos Aires. Años 2004-2016

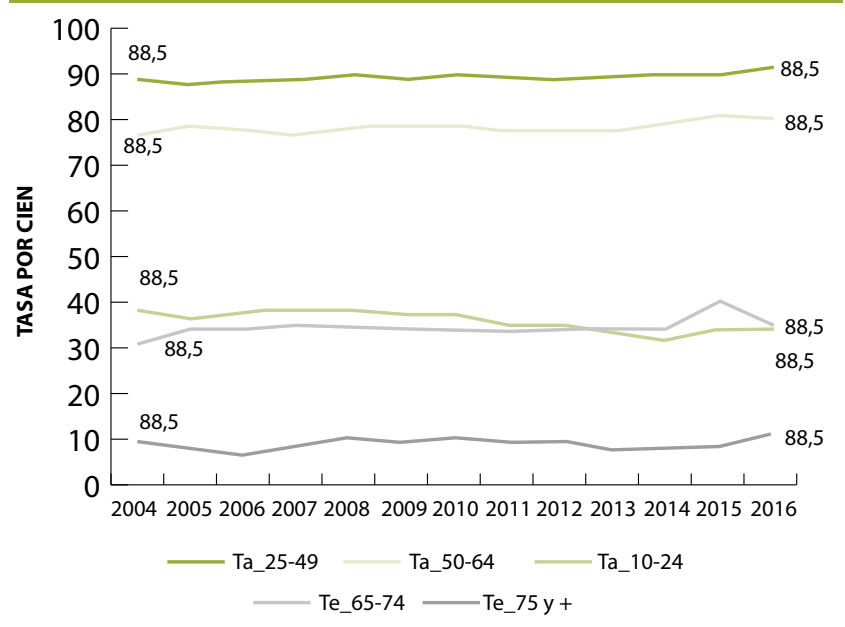

Fuente: Elaboración propia en base a datos EAH 2004-2016.

Ahora bien, ¿qué pasó con el empleo por grupo de edad? Las tasas de empleo en los menores de 25 años decrecen 6 puntos porcentuales. En el resto crece entre 3 puntos porcentuales (25-49 y 75 y + ), 4 puntos porcentuales (65-74 años) y 5 puntos porcentuales (50-64 años). En el último bienio se observa un incremento en la permanencia en el mercado de trabajo del grupo 65-74 años y en 2016 incluso de los mayores de esa edad (Gráfico №12).

El notable descenso del empleo de los menores de 25 años (31.8\% a 26.0\%) está condicionado por los efectos propios de la crisis y por la disminución de la población en dichas cohortes. Por su parte, el mantenimiento del empleo o incluso el aumento del mismo en determinados tramos de edad, entre los 50 y 74 años principalmente, se debe a que la población en dichas cohortes ha aumentado sensiblemente, especialmente por la incorporación de las mujeres al mercado laboral.

3 Por otra parte, la tasa correspondiente a varones prácticamente duplica la de las mujeres. 
Gráfico № 12 Tasas de empleo (por cien) según grupo de edad. Ciudad de Buenos Aires. Años 2004-2016

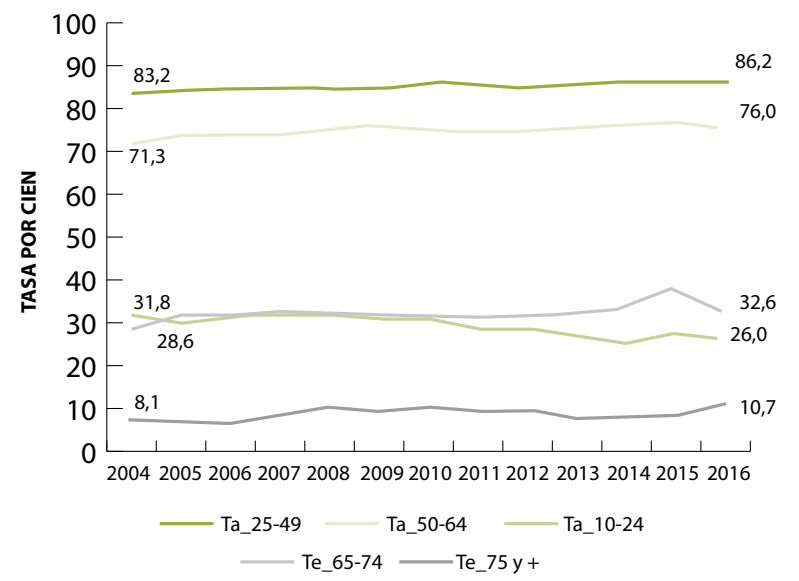

Fuente: Elaboración propia en base a datos EAH 2004/2016.

\subsection{Características de los adultos mayores que trabajaron en 2016}

A medida que la ciudad avanza en la transición demográfica, que la mortalidad y la fecundidad llegan a niveles bajos o muy bajos, el impacto directo en el crecimiento natural de la población se va haciendo cada vez más pequeño.

El efecto de estos cambios en la estructura por edad es de gran magnitud y se prolonga en el tiempo a medida que generaciones representativas de diferentes etapas del cambio demográfico avanzan en su trayectoria vital. El aumento del número de adultos mayores se suma al hecho de que las personas viven más años.

En la medida que este fenómeno se acelera, el proceso de envejecimiento y las relaciones de dependencia de las personas mayores empiezan a incrementarse, la relación numérica entre las personas en edades potencialmente dependientes (0-14 años y 65 años y más) y las personas en edades potencialmente activas ( 15 a 64 años) muestra claramente los efectos de la estructura por edades en una sociedad. El índice de dependencia en la ciudad de Buenos Aires asciende en el 2016 a 54.3 lo que significa que por cada 100 personas en edad de trabajar hay 54 personas en edades inactivas.

El envejecimiento poblacional, la reducción del tamaño medio de los hogares (2.4 en 2016) que tradicionalmente representan una red de seguridad y cuidados para los adultos mayores y los sistemas de seguridad social, a me- nudo insuficientes en términos de sostenibilidad financiera, tienen un efecto directo en el mercado de trabajo, que con la mejora de la esperanza de vida afectan al comportamiento individual en cuanto a la decisión de permanecer más tiempo en el trabajo.

Los mercados laborales, deberán adaptarse a la posibilidad de inserción de la población mayor de 60 años. Estos desafíos serán muy difíciles de resolver si no disminuyen los altos niveles de informalidad que caracterizan a los mercados laborales de la ciudad.

Los descriptores del mercado, muestran las características laborales de los adultos mayores ocupados y sus rasgos distintivos. En cuanto a la categoría ocupacional (Gráfico $N^{\circ} 13$ ) se destaca que en comparación con los menores de 65 años, entre los ocupados de la tercera edad hay menor proporción de asalariados y más del doble de población cuenta propia.

\section{Grafico Nº 13 Distribución porcentual de la población \\ ocupada por categoría ocupacional según grupo de edad. Ciudad de Buenos Aires. Añ̃o 2016}

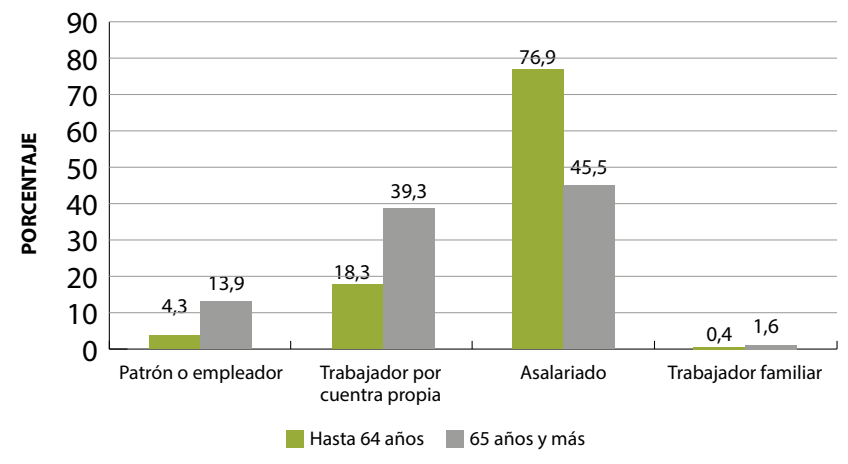

Fuente: Elaboración propia en base a datos EAH 2016.

Más de la mitad de la fuerza de trabajo que supera los 64 años desarrolla actividades en forma independiente, sólo $45.3 \%$ es asalariado.

En particular, el peso de los trabajadores por cuenta propia se duplica a partir de los 65 años $(39.3 \%$ frente al $18.3 \%$ para el resto de los ocupados). Por su parte, los patrones 0 empleadores se triplican: $4.3 \%$ en los menores de 65 años y $13.9 \%$ en los adultos mayores.

En comparación con el resto de los ocupados, en los mayores de 64 años, la tasa de asalarización ${ }^{4}$ se reduce 31.6 puntos porcentuales (Gráfico $\mathrm{N}^{\circ} 14$ ), mientras que la tasa de precarización ${ }^{5}$ (que se refiere a las características del 
puesto de trabajo), se duplica (de $21.8 \%$ a $52.1 \%$ ).

En cuanto a la tasa de registro ${ }^{6}$, en comparación, en los adultos mayores se reduce 25 puntos porcentuales, es decir algo más de la mitad de los adultos mayores asalariados, aportan por sí mismos o le descuentan para jubilación.

Se subraya que entre los ocupados de 65 años y más, hay mayor participación de mujeres que entre los ocupados menores de 65 años. Hay 84 mujeres de 65 años y más ocupadas por cada 100 varones ocupados de la misma franja etaria, valor que desciende a 65 en el resto de los ocupados.

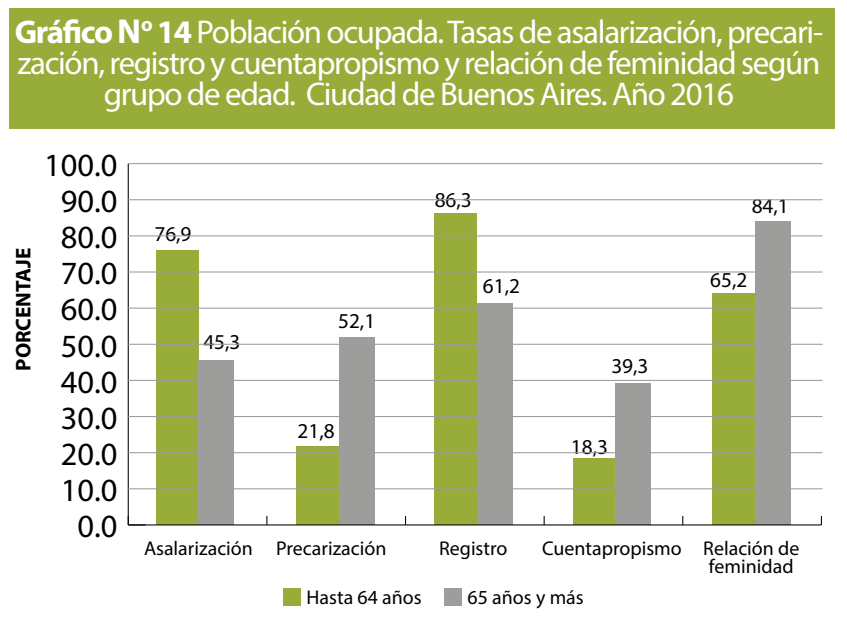

Fuente: Elaboración propia en base a datos EAH 2016.

La tendencia en los países desarrollados sugiere que, al aproximarse la edad de retiro, un número creciente de ocupados comienza a efectuar su transición hacia la inactividad a través de la reducción de la jornada laboral. Esta dinámica es poco marcada en los ocupados adultos mayores de la ciudad, que registran una jornada laboral promedio apenas una hora y media menor.

Como se observa en el Gráfico № 15 las horas promedio semanales de los varones ocupados se reducen particularmente a partir de los 75 años (12 horas semanales menos en promedio), mientras que en las mujeres este descenso es de sólo 7.6 horas semanales en promedio. Por lo tanto, a partir de los 75 años se acorta la brecha en las horas semanales trabajadas por varones y mujeres.

Otro aspecto interesante para analizar es la dinámica de las calificaciones ocupacionales, que tienen su explicación en la descripción de la distinta composición porcentual de las ocupaciones efectivamente desarrolladas según grupo de edad.

Gráfico № 15 Horas semanales trabajadas en la ocupación principal por sexo según grupo de edad. Ciudad de Buenos Aires. Año 2016

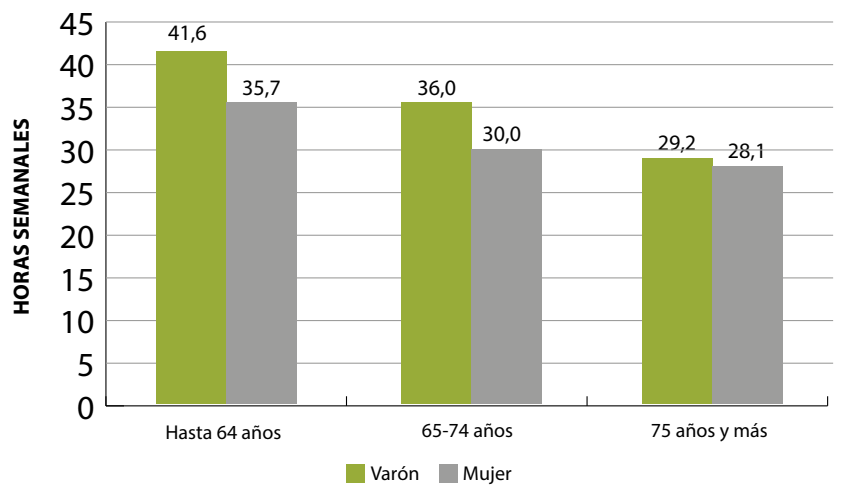

Fuente: Elaboración propia en base a datos EAH 2016.

Con respecto a la calificación ocupacional del puesto de trabajo ejercido, los adultos mayores tienen mayor participación los de calificación profesional y menor operativa, reafirmando la hipótesis de la disminución de los puestos de manualidad (Gráfico $N^{\circ} 16$ ). Los de 70 años y más registran calificación profesional en más del $30 \%$ de los ocupados, mientras que los menores alcanzan una cuarta parte. Por su parte, en la calificación operativa es lo contrario.

Gráfico № 16 Población ocupada de 25 años y más por calificacion ocupacional según grupo de edad. Ciudad de Buenos Aires. Año 2016

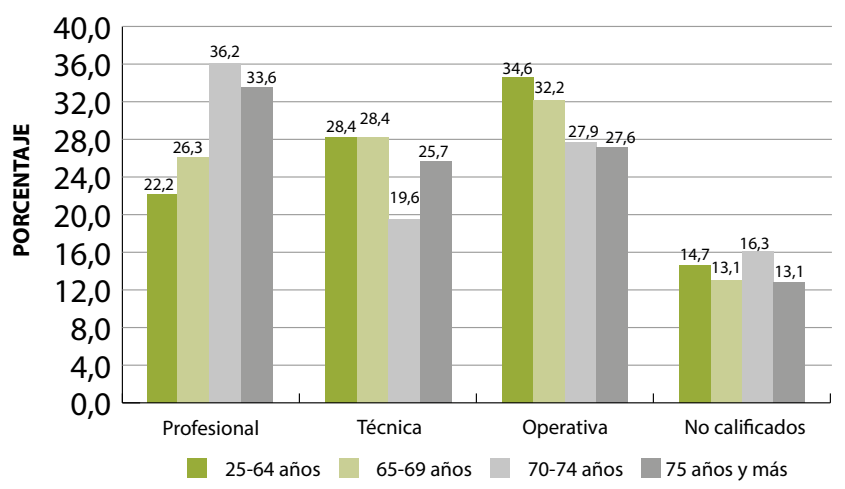

Fuente: Elaboración propia en base a datos EAH 2016.

La tecnología ocupacional del puesto de trabajo, establece la existencia de formas técnico-organizativas del trabajo, en cuyo contenido se revela la instalación, utilización y

por cien.

6 Población asalariada que aporta por sí misma o le descuentan para jubilación sobre el total de la población asalariada, por cien.

7 Cabe señalar que luego de la crisis de 2001 se produce la consolidación en la distribución de los sectores económicos de la ciudad y su consecuente modificación en los puestos de trabajo requeridos, adquiriendo preponderancia significativa el sector de servicios con un promedio de $86 \%$ en el período 2004-2016, sector que aporta cerca del 78\% del PGB de la ciudad. En una expresión cuantitativa, esta relación supone que, por cada ocupado ligado a ocupaciones productivas de bienes, existen alrededor de cinco que lo prestan en el sector servicios. 
mantenimiento de maquinaria y equipos, la investigación y desarrollo de productos y procesos ${ }^{8}$.

Como se observa en el Gráfico №17, en la población de la Ciudad existen diferencias importantes según grupo de edad en la tecnología ocupacional. Aproximadamente el $50 \%$ de los ocupados de 65 años y más, utilizan instrumentos simples o ejecutan directamente prestación de un servicio.
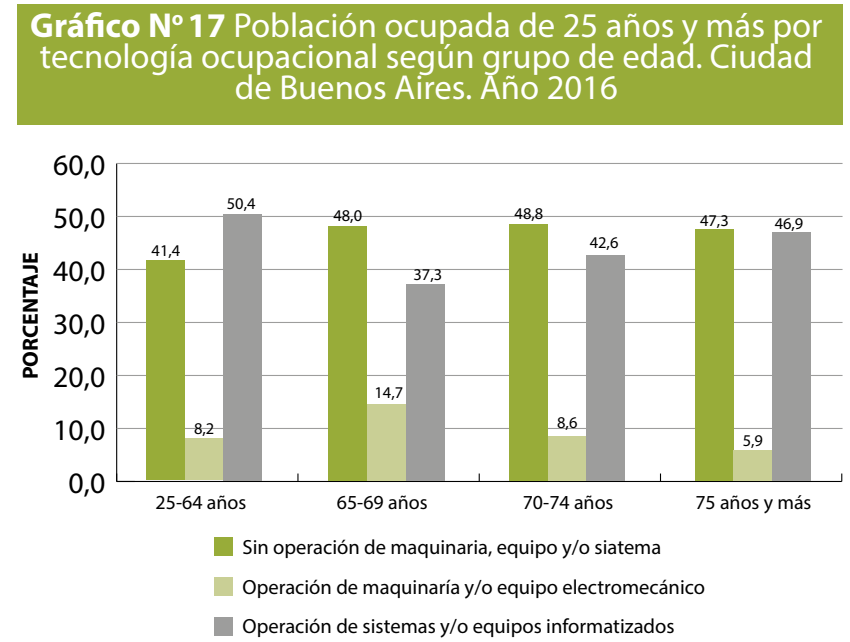

Fuente: Elaboración propia en base a datos EAH 2016.

Entre los adultos mayores, las ocupaciones con operación de maquinaria decrecen con la edad. Por su parte, las ocupaciones que utilizan equipos informatizados aumentan según las distintas franjas etarias de los adultos mayores.

Se destaca que a partir de los 70 años se reduce a la mitad la operación de maquinaria y/o equipos electromecánicos.

\subsection{Los ingresos no laborales y la necesidad de permanecer en el mercado de trabajo}

Como ya se expresará, los ingresos constituyen un factor significativo con fines de permanecer o retirarse del mercado de trabajo productivo. La cobertura de jubilaciones y pensiones, cercana a $93 \%$ en los de 65 años y más, cuyo monto se actualiza trimestralmente, garantiza un mínimo de ingresos para los hogares en los que estas personas residen y resulta en una incidencia de la pobreza inferior a un tercio de la correspondiente al conjunto de la ciudad.

Se destaca que el porcentaje de las mujeres de 65 años y más con cobertura previsional en la ciudad se encuentra cercano al 100\% (93.7\% según datos del Censo 2010), mientras que sus pares varones registran el $87.8 \%$.
Más de la mitad de los hogares con presencia de adultos mayores se encuentra en los quintiles 2 y 3 de la distribución del ingreso per cápita familiar. En el $21.6 \%$ de los hogares en los que solo residen adultos mayores, los ingresos provienen simultáneamente del trabajo y de otras fuentes (especialmente jubilaciones y pensiones, pero también de otras transferencias y de ingresos provenientes de la propiedad de activos), lo que muestra nuevamente que, para una parte significativa de esta población, el trabajo sigue teniendo centralidad más allá de que sean perceptores de jubilaciones y pensiones.

\section{Conclusiones}

Entonces ¿qué papel jugaron las mujeres en el contexto del envejecimiento de la población activa en la ciudad de Buenos Aires? Puede afirmarse que la feminización del envejecimiento de la población, el aumento de la participación económica de las mujeres desde fines del siglo pasado y su permanencia en el mercado de trabajo hasta edades que superan el retiro, incidieron en el envejecimiento de la PEA de la ciudad.

El incremento en la participación laboral femenina responde a una amplia gama de factores. A partir de los años sesenta se asistió a la redefinición del papel de la mujer en la sociedad, en las relaciones de género y en la institución familiar. El matrimonio empezó a dejar de ser visto como un mero ámbito de reproducción. Las mujeres comenzaron a aumentar su participación laboral, a un ritmo lento hasta los sesenta y más acelerado en las décadas siguientes. Por otro lado, se relaciona con el desigual aumento de la tasa de actividad económica entre varones y mujeres.

El artículo muestra que el envejecimiento de la población total se ha traslado a la PEA y que este envejecimiento está relacionado con el aumento de la población en edad de trabajar y la población activa femenina.

Mientras que el volumen de los varones activos entre 2002 y 2016 creció el 16\%; las mujeres activas, en igual período lo hicieron el $23 \%$. Por otro lado, el volumen máximo de la PEA masculina se registró en 2007 y a partir de ese momento ha ido descendiendo hasta estancarse. Por su parte el guarismo de la PEA femenina ha ido creciendo, con pequeñas oscilaciones, hasta registrar el máximo en 2016.

8 Este nomenclador reconoce cuatro categorías: 1) Ocupaciones sin operación de maquinaria, equipos y sistemas: en las cuales se utilizan sólo instrumentos simples y/o la habilidad física o intelectual, o se ejecuta directamente la producción de un bien o la prestación de un servicio; 2) Ocupaciones con operación de maquinaria, equipos o sistemas electromecánicos: las que necesitan para su ejercicio la operación constante de este tipo de instrumentos; 3) Ocupaciones con operación de equipos y/o sistemas informatizados: en las cuales se utiliza en forma necesaria y constante instrumental informatizado y 4) Ocupaciones del desarrollo tecnológico, la instalación y la reparación de maquinaria y/o equipos: en las que se realiza la instalación y mantenimiento de maquinaria, equipos y sistemas; la investigación y desarrollo de productos y procesos. 
El envejecimiento de la PEA alteró el mercado de trabajo. Las tasas de actividad y de empleo de los menores de 25 años se redujeron. Las de la población de 65 años y más aumentaron, especialmente en las mujeres.

También los descriptores del mercado de trabajo de los adultos mayores son diferentes a los de menores de 65 años: más cuentapropistas, menor brecha horaria entre varones y mujeres de horas de trabajo, mayor calificación profesional y menor operativa.

Salvo que se produzcan notables variaciones en los flujos migratorios, las proyecciones apuntan a un estancamiento e inclusive descenso de la población y a un notable envejecimiento de la misma para los próximos años. Este envejecimiento de la población total conllevará el descenso y envejecimiento de la población activa, a pesar del repunte de las tasas de actividad que se produzca por la recuperación de la economía y la incorporación de la mujer al mercado de trabajo. Por otro lado, están las implicancias que puede tener para la relación entre ocupados y jubilados en el mediano plazo, es decir en la sostenibilidad del sistema de seguridad social.

El proceso de envejecimiento futuro de la población de la ciudad de Buenos Aires condicionará la evolución futura de la población activa. El impacto del envejecimiento ralentizará el crecimiento económico. Habrá menos trabajadores potenciales por cada ciudadano. El envejecimiento modificará el tipo de ocupaciones más demandadas. Ellas serán: servicios médicos, de cuidados y atención a personas mayores. El envejecimiento incrementará los costos de salud y seguridad social.

Cambios todos ellos en los que es necesario comenzar a reflexionar e incluso plantear soluciones lo antes posible. Desafíos difíciles de resolver si no disminuyen los altos niveles de informalidad que caracterizan los mercados laborales de la región. A lo que debe agregarse las transformaciones que surgen del cambio tecnológico, ya que la incorporación de tecnología genera retos de adaptación, de modernización y reducción de riesgos.

El futuro del trabajo estará marcado por estas dos grandes tendencias: el avance tecnológico y el envejecimiento poblacional.

\section{Referencias bibliográficas}

- Banco Interamericano de Desarrollo (2018). El futuro del trabajo en América Latina y el Caribe. ¿Una gran oportunidad para la región? Disponible en: www.iadb.org/ futurodeltrabajo.
- Comelatto, Pablo A. (2009). Cambio demográfico en la Ciudad de Buenos Aires y sus relaciones con el cambio en la participación económica. Población de Buenos Aires. año 6. no 9. (pp. 23-28). Ciudad de Buenos Aires.

- Ham-Chande, Roberto, Alberto Palloni y Rebeca Wong (2009). El envejecimiento en países en desarrollo: estableciendo lazos para integrar agendas de investigación. Documentos de políticas de investigación n² 22 . France. IUSSP.

- Laville, Antoine (1993). Envejecimiento de las funciones fisiológicas y cognitivas y trabajo. En Laville, Antoine (Coord.) Envejecimiento y trabajo. Buenos Aires, Trabajo y Sociedad - PIETTE/CONICET - CEIL/CONICET - CREDAL/ CNRS.

- Lattes, Alfredo E. y Gretel Andrada (2006a). Subsistema demográfico de la Ciudad de Buenos Aires: dinámica de la población económicamente activa entre 1950 y 2000. Población de Buenos Aires. año 3. no 3. (pp. 67-87). Ciudad de Buenos Aires.

- Lattes, Alfredo E., Gretel Andrada y Julieta Vera (2006b). Subsistema demográfico de la Ciudad de Buenos Aires: población y trabajo. Población de Buenos Aires. año 3. no 4. (pp. 77-89). Ciudad de Buenos Aires.

- Lattes, Alfredo E., Gretel Andrada y Pablo Caviezel (2010). Dinámica demográfica. En Dirección General de Estadística y Censos. Dinámica de una ciudad. Buenos Aires, 18102010. (pp. 129-164). Gobierno de la Ciudad de Buenos Aires.

- Mazzeo, Victoria (2017). La vejez de las mujeres en Argentina. ¿En qué se parecen y en qué difieren las adultas mayores de ayer y de hoy? en XII Jornadas de Sociología. Disponible en: http://jornadasdesociologia2017.sociales. uba.ar/wp-content/uploads/ponencia/343_195.pdf

- Pérez Díaz, J. (2003). La Madurez de masas. Madrid: Instituto de Migraciones y Sevicios Sociales.

- Segalen, Martine (2013). Sociología de la familia. EUDEM. Mar del Plata. 7ma edición. 